\title{
Event-based robust stabilization for delayed systems with parameter uncertainties and exogenous disturbances
}

\section{Yuwei Du' and Bing $\mathrm{Li}^{1 *}$ (B)}

"Correspondence:

libingcnjy@163.com

${ }^{1}$ The School of Mathematics and

Statistics, Chongqing Jiaotong

University, Chongqing, China

\section{Springer}

\begin{abstract}
In this paper, the robust stabilization problem is studied for a class of delayed systems with parameter uncertainties and unknown-but-bounded exogenous disturbances. The robust input-to-state practical stability (RISpS) is introduced to characterize the dynamics of the controlled system. An event-triggered strategy is employed to effectively decrease the transmission consumption of the robust controller. The Zeno behavior is also excluded by combining the information of delayed states with parameter uncertainties. The gain matrix and the event-triggered parameters are co-designed by resorting to the feasibility of several matrix inequalities. An example and its simulations are given to illustrate the proposed approach.
\end{abstract}

Keywords: Uncertain delayed system; Input-to-state stability; Event-triggered control; Zeno behavior

\section{Introduction}

The arising of time delays in control systems is inevitable due to the finite switching speed of information storage processing, communication time and transmission of signals. As is well known, the time delays can influence the dynamic of systems seriously and may cause some unstable performances such as divergence, oscillation or chaotic. Over the past few years, a great number of research papers have been published to focus on the stability and stabilization of dynamical systems with time delays (see [1-6] and the references cited therein). At the same time, it should be mentioned that under some exogenous disturbances, the system states may evolve in a bounded domain rather than converge to an equilibrium point, which indicates that the conventional stability cannot be realized. Inspired by Sontag [7], the input-to-state stability (ISS) can effectively characterize the robustness of stability over exogenous disturbances. In recent years, the ISS properties for delayed systems have gained the rapidly growing research interest from control community [8-15].

Moreover, control systems usually encounter a variety of uncertainties resulting from the inaccuracy of physical parameters, quantization errors, and unmolded factors. During the implementation, the parameter fluctuation and errors also lead to the poor performance and instability of closed-loop systems. Hence the uncertain parameters of a system

(c) The Author(s) 2020. This article is licensed under a Creative Commons Attribution 4.0 International License, which permits use sharing, adaptation, distribution and reproduction in any medium or format, as long as you give appropriate credit to the original author(s) and the source, provide a link to the Creative Commons licence, and indicate if changes were made. The images or other third party material in this article are included in the article's Creative Commons licence, unless indicated otherwise in a credit line to the material. If material is not included in the article's Creative Commons licence and your intended use is not permitted by statutory regulation or exceeds the permitted use, you will need to obtain permission directly from the copyright holder. To view a copy of this licence, visit http://creativecommons.org/licenses/by/4.0/. 
are only due to the deviations and perturbations of its parameters. Recently, significant progress has been made in the study of robust stability and controller synthesis for delayed systems with parameter uncertainties [16-27]. For example, in [17], the robust stability has been studied for a class of linear networked control systems with dynamic quantization, variable sampling intervals as well as communication delays. In [27], the passivity analysis has been studied for uncertain BAM neural networks with leakage, discrete and distributed delays with the help of novel summation inequality. By employing the L-K functional method, authors have investigated the robust exponential stability problem for a class of uncertain inertial type BAM neural networks with of mixed delays in [21]. It is noted that most literature mentioned above has focused on the stability of delayed systems with uncertainties. So far, very little attention has been paid to the ultimately bounded performances for uncertain delayed systems under some exogenous disturbances. Hence, it is also of significant importance to address the robust input-to-state stability (RISS) for delayed systems with parameter uncertainties and exogenous disturbances, which is the first motivation of this research.

In many computer- and network-based control systems, the control signals can only be transmitted at discrete instants because of the implement of digital platforms. As such, the event-triggered control mechanisms, in which the control signals are updated when a certain event occurs, are often employed to avoid the unnecessary consumption of resources while maintaining the expected control performance [28]. In last decade, the event-triggered strategy has been extensively adopted for engineering applications including asymptotical stability [29-33], ISS [34-36], state estimation [37-39], consensus analysis [40, 41], and nonlinear control [42-46]. However, the corresponding researches on the event-triggered RISS for uncertain delayed systems have been relatively scattered despite their great significance in practical applications, and this constitutes the second motivation for us to carry out this paper.

Motivated by the above discussions, we aim to investigate the robust input-to-state practical stability (RISpS) and the corresponding event-triggered controller design for a kind of uncertain delayed systems with unknown-but-bounded exogenous disturbances. The main advantages of this paper are summarized by the following three aspects:

(1) The RISpS property is, for the first time, proposed to evaluate the dynamical behaviors for a class of uncertain delayed systems with disturbances, which is quite different from and more comprehensive than the ISS properties investigated in $[7-10,12,13,15,34-36]$ and the robust stability studied in [16-27].

(2) Several previous literature such as $[1,3,6,11,23,27]$ required that the control signal updates continuously with time, which may lead to the unnecessary cost of network resources. In this paper, the event-triggered scheme is introduced to generates a sporadic control sequence while maintaining the desired ISpS dynamical performance for all admissible uncertainties. Hence, this co-design control framework can improve overall control system performance while reducing the real-time system's use of computational resources.

(3) Those methods proposed in existing literature [29-32,34] are invalid for analyzing the Zeno behavior because of the presence of time delays and parameter uncertainties. In this paper, with the help of delayed differential inequality and impulsive jumping estimation techniques, the associated Zeno phenomenon is 
effectively excluded for the proposed event-triggered scheme by integrating the

information of current state, time delays, disturbances, and parameter uncertainties.

The rest of this paper is organized as follows. In Sect. 2, the mathematical model and several preliminaries are presented. The problems of RISpS analysis and event-triggered controller design are solved in Sect. 3. A numerical example is given in Sect. 4 to illustrate the effectiveness of theoretical results. Finally, Sect. 5 concludes our research.

Notations. Let $\mathbb{R}_{0}^{+}$be the set of nonnegative real numbers and $\mathbb{Z}_{0}^{+}$be the set of nonnegative integers. $\mathbb{R}^{n}$ stands for the $n$-dimensional Euclidean space and $\mathbb{R}^{n \times m}$ represents the class of $n \times m$ real matrices. $|x|$ and $\|A\|$ denote the Euclidean vector norm of $x$ and the induced matrix norm, respectively. $I$ is for the identity matrix with compatible dimension. The symbol $*$ indicates a symmetric structure in matrix expressions. $\lambda_{\min }(\cdot)$ and $\lambda_{\max }(\cdot)$ refer to the smallest and the largest eigenvalue of a symmetric matrix, respectively. Denote by $\mathcal{L}_{\infty}^{n}$ the class of measurable and essentially bounded functions $v: \mathbb{R}_{0}^{+} \rightarrow \mathbb{R}^{n}$ with the infinity norm $|v|_{\infty}:=\operatorname{ess~sup~}_{t \in \mathbb{R}_{0}^{+}}\{|v(t)|\}<\infty$. A continuous function $\gamma: \mathbb{R}_{0}^{+} \rightarrow \mathbb{R}_{0}^{+}$is a $\mathcal{K}$-function if it is strictly increasing and $\gamma(0)=0$; it is a $\mathcal{K}_{\infty}$-function if it is a $\mathcal{K}$-function and satisfies $\gamma(s) \rightarrow \infty$ as $s \rightarrow \infty$. A function $\beta: \mathbb{R}_{0}^{+} \times \mathbb{R}_{0}^{+} \rightarrow \mathbb{R}_{0}^{+}$is a $\mathcal{K} \mathcal{L}$-function if for each fixed $t \in \mathbb{R}_{0}^{+}, \beta(\cdot, t)$ is a $\mathcal{K}$-function, and for each fixed $s \in \mathbb{R}_{0}^{+}, \beta(s, \cdot)$ is decreasing and $\beta(s, t) \rightarrow 0$ as $t \rightarrow \infty$. For $\tau>0, \mathcal{C}\left([-\tau, 0] ; \mathbb{R}^{n}\right)$ denotes the class of all continuous $\mathbb{R}^{n}$-value functions $\varphi$ on $[-\tau, 0]$ with norm $|\varphi|_{\tau}:=\sup \{|\varphi(s)|:-\tau \leq s \leq 0\} . \mathcal{B}(0, r):=\left\{x \in \mathbb{R}^{n}:|x| \leq r\right\}$ for $r \geq 0$.

\section{Model and preliminaries}

Consider a class of uncertain delayed systems with unknown-but-bounded exogenous disturbance as follows:

$$
x^{\prime}(t)=(A+\Delta A) x(t)+\left(A_{d}+\Delta A_{d}\right) x_{\tau}(t)+B u(t)+C v(t), \quad t \geq 0,
$$

where $x(t) \in \mathbb{R}^{n}, x_{\tau}(t):=x(t-\tau(t))$ with $0 \leq \tau(t) \leq \tau$ are the states, $u(t) \in \mathbb{R}^{q}$ is control input, $v(t) \in \mathcal{L}_{\infty}^{n}$ is exogenous disturbance. $A, A_{d}, B, C$ are known constant matrices with compatible dimensions. $\Delta A, \Delta A_{d}$ are for parameter uncertainties satisfying

$$
\Delta A=E_{1} F_{1}(t) H_{1}, \Delta A_{d}=E_{2} F_{2}(t) H_{2}
$$

in which $F_{i}^{T}(t) F_{i}(t) \leq I$ and $E_{i}, H_{i}(i=1,2)$ are known constant matrices.

In this paper, we assume that the system state is sampled first based on an eventtriggered mechanism and then transmitted to the actuator in the zeroth-order hold ( $\mathrm{ZOH})$ fashion. In other words,

$$
u(t)=K x\left(t_{i}\right), \quad t \in\left[t_{i}, t_{i+1}\right), i \in \mathbb{Z}_{0}^{+} .
$$

Here, $\left\{t_{i}: i \in \mathbb{Z}_{0}^{+}\right\}$denotes the triggering instant sequence which is determined iteratively by

$$
t_{i+1}=\inf _{t}\left\{t>t_{i}:\left|x\left(t_{i}\right)-x(t)\right|^{2} \geq \xi_{1}\left|x\left(t_{i}\right)\right|^{2}+\xi_{2}\right\}
$$

in which the positive constants $\xi_{1}, \xi_{2}$ denote the weight and threshold parameters, respectively. 
Remark 1 For a control system operated on the digital platform, the periodic sampling and transmission of data is advantageous from the design standpoint but may lead to higher system costs. The event-triggered control, which means that the control task is only executed when the application's error signal exceeds a specified threshold, will generate a much lower updating frequency of sporadic control sequence. There has been experimental evidence to support the assertion that event-triggered feedback improves overall control system performance while reducing the real-time system's use of computational resources [31]. This co-design control framework has been widely used to address the problem of scheduling stabilizing control tasks on embedded processors [33, 38], information fusions [39], the average consensus of multi-agent systems [31, 40, 41].

Within the event-triggered controller, the closed-loop system is obtained as follows:

$$
x^{\prime}(t)=(A+\Delta A) x(t)+\left(A_{d}+\Delta A_{d}\right) x_{\tau}(t)+B K x\left(t_{i}\right)+C v(t)
$$

for $t \in\left[t_{i}, t_{i+1}\right), i \in \mathbb{Z}_{0}^{+}$. By denoting the measurement error (between the current state and sampled state)

$$
e(t)=x\left(t_{i}\right)-x(t)
$$

we have

$$
x^{\prime}(t)=(A+\Delta A+B K) x(t)+\left(A_{d}+\Delta A_{d}\right) x_{\tau}(t)+B K e(t)+C v(t) .
$$

Remark 2 The control model (7) is more comprehensive than those studied in previous literature $[6,9,10,13,35,36,44]$ because of the simultaneous presence of parameter uncertainties, time-varying delays, and the bounded disturbances.

The following definition and lemmas will be useful in later discussion.

Definition 1 The closed-loop system (7) is said to be robustly input-to-state practically stable if there are functions $\beta_{c} \in \mathcal{K} \mathcal{L}, \gamma_{c} \in \mathcal{K}$ and a scalar $d_{c} \in \mathbb{R}_{0}^{+}$such that

$$
|x(t)| \leq \beta_{c}\left(|\varphi|_{\tau}, t\right)+\gamma_{c}\left(|v|_{\infty}\right)+d_{c}
$$

for any $t \in \mathbb{R}_{0}^{+}, \varphi \in \mathcal{C}\left([-\tau, 0] ; \mathbb{R}^{n}\right), v \in \mathcal{L}_{\infty}^{n}$ and all parameter uncertainties satisfying (2).

Remark 3 By taking into account both parameter uncertainties and exogenous disturbances, Definition 1 proposes a novel and more practical perspective to analyze the dynamics of system (7). When all uncertainties are removed, Definition 1 reduces to the conventional ISS concept introduced in $[11,12,34]$ and the term $\gamma_{c}\left(|v|_{\infty}\right)+d_{c}$ is used to represent the bound of the domain where the state remains. When $d_{c}=0$ and $v(t) \equiv 0$, Definition 1 reduces to the asymptotically robust stability considered in [20-22, 24-26] and the $\mathcal{K} \mathcal{L}$-function $\beta_{c}$ indicates that the state will tend to zero as $t \rightarrow+\infty$ for all admissible parameter uncertainties satisfying (2). 
Lemma 1 For any $x, y \in \mathbb{R}^{n}$,

$$
x^{T} y+y^{T} x \leq x^{T} Q x+y^{T} Q^{-1} y
$$

where $Q$ is a positive definite matrix with appropriate dimension.

Lemma 2 ([47]) Let $P \in \mathbb{R}^{n \times n}$ be a symmetric matrix and $x \in \mathbb{R}^{n}$ be a vector. Then we have

$$
\lambda_{\min }(P) x^{T} x \leq x^{T} P x \leq \lambda_{\max }(P) x^{T} x .
$$

Lemma 3 ([48]) Assume that $A, D, G, F, W>0$ are matrices with appropriate dimensions and $F^{T} F \leq I$. For any scalar $\varepsilon_{1}>0$,

$$
2 x^{T} D F G y \leq \varepsilon_{1}^{-1} x^{T} D D^{T} x+\varepsilon_{1} y^{T} G^{T} G y .
$$

If there is a scalar $\varepsilon_{2}>0$ such that

$$
W-\varepsilon_{2} D D^{T}>0
$$

then

$$
(A+D F G)^{T} W^{-1}(A+D F G) \leq A^{T}\left(W-\varepsilon_{2} D D^{T}\right)^{-1} A+\varepsilon_{2}^{-1} G G^{T} .
$$

\section{Main results}

In this section, a theoretical framework is established to analyze the RISpS for the uncertain delayed system under consideration. Moreover, the Zeno behavior is discussed and excluded for the proposed event-triggered strategy via integrating the information of current and delayed states, parameter uncertainties, and the exogenous disturbances.

Theorem 1 Let $K \in \mathbb{R}^{q \times n}$ and constants $\xi_{1} \in\left(0, \frac{1}{2}\right), \xi_{2}>0$ be given. If there exist positive definite matrices $P \in \mathbb{R}^{n \times n}, Q \in \mathbb{R}^{n \times n}$ and four positive scalars $\varepsilon_{i}(i=1,2,3,4)$ such that

$$
\begin{aligned}
& I-\varepsilon_{2} E_{2} E_{2}^{T}>0, \\
& \Pi_{1}+\varepsilon_{3} P<0, \\
& \Pi_{2}-\varepsilon_{4} P<0, \\
& \varepsilon_{3}-\varepsilon_{4}-\frac{2 \xi_{1} \lambda_{\max }\left(P^{2}\right)}{\left(1-2 \xi_{1}\right) \lambda_{\min }(P)}>0,
\end{aligned}
$$

where $\Pi_{1}=P(A+B K)+(A+B K)^{T} P+P C Q^{-1} C^{T} P+P^{2}+\varepsilon_{1} H_{1}^{T} H_{1}+\varepsilon_{1}^{-1} P E_{1} E_{1}^{T} P+$ $P B K P^{-2} K^{T} B^{T} P, \Pi_{2}=A_{d}^{T}\left(I-\varepsilon_{2} E_{2} E_{2}^{T}\right)^{-1} A_{d}+\varepsilon_{2}^{-1} H_{2}^{T} H_{2}$, then the closed-loop system (7) is robustly input-to-state practically stable with respect to all parameter uncertainties satisfying (2).

Proof We choose a Lyapunov function as follows:

$$
V(t):=V(x(t))=x^{T}(t) P x(t) .
$$


By calculating the derivative of $V(t)$ along system (7), one has

$$
\begin{aligned}
V^{\prime}(t)= & 2 x^{T}(t) P(A+\Delta A+B K) x(t)+2 x^{T}(t) P B K e(t) \\
& +2 x^{T}(t) P\left(A_{d}+\Delta A_{d}\right) x_{\tau}(t)+2 x^{T}(t) P C v(t) .
\end{aligned}
$$

It follows from Lemma 1 and Lemma 3 that

$$
\begin{aligned}
2 x^{T}(t) P(A+\Delta A+B K) x(t) \leq & x^{T}(t)\left[P(A+B K)+(A+B K)^{T} P\right. \\
& \left.+\varepsilon_{1}^{-1} P E_{1} E_{1}^{T} P+\varepsilon_{1} H_{1}^{T} H_{1}\right] x(t)
\end{aligned}
$$

and

$$
\begin{aligned}
2 x^{T}(t) P\left(A_{d}+\Delta A_{d}\right) x_{\tau}(t) \leq & x^{T}(t) P^{2} x(t)+x_{\tau}^{T}(t)\left[\varepsilon_{2}^{-1} H_{2}^{T} H_{2}\right] x_{\tau}(t) \\
& +x_{\tau}^{T}(t) A_{d}^{T}\left(I-\varepsilon_{2} E_{2} E_{2}^{T}\right)^{-1} A_{d} x_{\tau}(t) .
\end{aligned}
$$

According to Lemma 1 and Lemma 2, we have

$$
\begin{aligned}
2 x^{T}(t) P C v(t) & \leq x^{T}(t) P C Q^{-1} C^{T} P x(t)+v^{T}(t) Q v(t) \\
& \leq x^{T}(t) P C Q^{-1} C^{T} P x(t)+\lambda_{\max }(Q)|v(t)|^{2}
\end{aligned}
$$

and

$$
\begin{aligned}
2 x^{T}(t) P B K e(t) & \leq x^{T}(t) P B K\left(P^{T} P\right)^{-1} K^{T} B^{T} P x(t)+e^{T}(t)\left(P^{T} P\right) e(t) \\
& \leq x^{T}(t) P B K P^{-2} K^{T} B^{T} P x(t)+\lambda_{\max }\left(P^{2}\right)|e(t)|^{2} .
\end{aligned}
$$

Substituting (14)-(17) into (13) gives

$$
V^{\prime}(t) \leq x^{T}(t) \Pi_{1} x(t)+x_{\tau}^{T}(t) \Pi_{2} x_{\tau}(t)+\lambda_{\max }(Q)|v(t)|^{2}+\lambda_{\max }\left(P^{2}\right)|e(t)|^{2}
$$

which, together with (9) and (10), implies that

$$
\begin{aligned}
V^{\prime}(t) \leq & -\varepsilon_{3} x^{T}(t) P x(t)+\varepsilon_{4} x_{\tau}^{T}(t) P x_{\tau}(t) \\
& +\lambda_{\max }\left(P^{2}\right)|e(t)|^{2}+\lambda_{\max }(Q)|v(t)|^{2} .
\end{aligned}
$$

It is worth noting that the measurement error $e(t)$ is subjected to the constraint of the event-triggered rule (4). Thus, one derives

$$
|e(t)|^{2} \leq 2 \xi_{1}\left(|e(t)|^{2}+|x(t)|^{2}\right)+\xi_{2}
$$

which further leads to

$$
|e(t)|^{2} \leq \frac{2 \xi_{1}}{1-2 \xi_{1}}|x(t)|^{2}+\frac{\xi_{2}}{1-2 \xi_{1}} .
$$


Bearing in mind that $V(t) \geq \lambda_{\min }(P)|x(t)|^{2}$, we get

$$
|e(t)|^{2} \leq \frac{2 \xi_{1}}{\left(1-2 \xi_{1}\right) \lambda_{\min }(P)} V(t)+\frac{\xi_{2}}{1-2 \xi_{1}}
$$

By combining (21) with (19), one obtains

$$
V^{\prime}(t) \leq-\bar{\varepsilon}_{3} V(t)+\varepsilon_{4} V(t-\tau(t))+d+\lambda_{\max }(Q)|v(t)|^{2}, \quad t \geq 0
$$

where $\bar{\varepsilon}_{3}=\varepsilon_{3}-\frac{2 \lambda_{\max }\left(P^{2}\right) \xi_{1}}{\left(1-2 \xi_{1}\right) \lambda_{\min }(P)}, d=\frac{\lambda_{\max }\left(P^{2}\right) \xi_{2}}{1-2 \xi_{1}}$.

We consider the function $h(t)=t-\bar{\varepsilon}_{3}+\varepsilon_{4} e^{\tau t}$ for $t \geq 0$. It is easy to calculate that $h^{\prime}(t)=$ $1+\varepsilon_{4} \tau e^{\tau t}>0$, which indicates that $h(t)$ is a monotonically increasing function. From (11), one gets $h(0)=-\bar{\varepsilon}_{3}+\varepsilon_{4}<0$ and $\lim _{t \rightarrow+\infty} h(t)=+\infty$. Hence, there is a unique positive scalar $\varrho^{*}$ such that

$$
\varrho^{*}-\bar{\varepsilon}_{3}+\varepsilon_{4} e^{\varrho^{*} \tau}=0
$$

For any given $\varrho \in\left(0, \varrho^{*}\right]$ and non-zero initial function $\varphi \in \mathcal{C}\left([-\tau, 0] ; \mathbb{R}^{n}\right)$, we construct the following functions:

$$
U^{*}(t)=e^{\varrho t} V(t), \quad t \in[-\tau,+\infty)
$$

and

$$
U(t)= \begin{cases}\lambda_{\max }(P)|\varphi|_{\tau}^{2}, & t \in[-\tau, 0), \\ \lambda_{\max }(P)|\varphi|_{\tau}^{2}+\int_{0}^{t} e^{\varrho s}\left(d+\lambda_{\max }(Q)|v(s)|^{2}\right) d s, & t \geq 0\end{cases}
$$

In what follows, we will verify

$$
U^{*}(t) \leq U(t), \quad t \geq 0
$$

For $t \in[-\tau, 0)$, it is readily concluded that

$$
U^{*}(t)=e^{\varrho t} V(t)<V(t) \leq \lambda_{\max }(P)|\varphi(t)|^{2} \leq U(t) .
$$

If (25) does not hold, then there exists some $t>0$ such that $U^{*}(t)>U(t)$. By denoting $t^{*}:=\inf _{t}\left\{t>0: U^{*}(t)>U(t)\right\}$, it is derived from the continuity of $U^{*}(t)$ and $U(t)$ that

$$
\begin{aligned}
& U^{*}(t)<U(t), \quad t \in\left[-\tau, t^{*}\right), \\
& U^{*}\left(t^{*}\right)=U\left(t^{*}\right)
\end{aligned}
$$

and there is a sufficiently small positive scalar $\Delta t^{\prime}$ such that

$$
U^{*}(t)>U(t), \quad t \in\left(t^{*}, t^{*}+\Delta t^{\prime}\right) .
$$


Calculating the upper right-hand Dini derivative of $U^{*}(t)$ at $t^{*}$ yields

$$
\begin{aligned}
D^{+} U^{*}\left(t^{*}\right) & :=\limsup _{h \rightarrow 0^{+}} \frac{U^{*}\left(t^{*}+h\right)-U^{*}\left(t^{*}\right)}{h} \\
& \geq \limsup _{h \rightarrow 0^{+}} \frac{U\left(t^{*}+h\right)-U\left(t^{*}\right)}{h} \\
& =e^{\varrho t^{*}}\left(d+\lambda_{\max }(Q)\left|v\left(t^{*}\right)\right|^{2}\right) .
\end{aligned}
$$

On the other hand, it is readily concluded from (22) and (23) that

$$
\begin{aligned}
D^{+} U^{*}\left(t^{*}\right)= & \varrho e^{\varrho t^{*}} V\left(t^{*}\right)+e^{\varrho t^{*}} D^{+} V\left(t^{*}\right) \\
\leq & \left(\varrho-\bar{\varepsilon}_{3}\right) e^{\varrho t^{*}} V\left(t^{*}\right)+\varepsilon_{4} e^{\varrho t^{*}} V\left(t^{*}-\tau\left(t^{*}\right)\right) \\
& +d e^{\varrho t^{*}}+\lambda_{\max }(Q) e^{\varrho t^{*}}\left|v\left(t^{*}\right)\right|^{2} .
\end{aligned}
$$

Noting that $U(t)$ is a monotone nondecreasing function on $[-\tau,+\infty)$, we derive

$$
U^{*}\left(t^{*}-\tau\left(t^{*}\right)\right)<U\left(t^{*}-\tau\left(t^{*}\right)\right)<U\left(t^{*}\right)=U^{*}\left(t^{*}\right)
$$

which further implies

$$
V\left(t^{*}-\tau\left(t^{*}\right)\right)<e^{\varrho \tau} V\left(t^{*}\right)
$$

It should be observed that $\varrho-\bar{\varepsilon}_{3}+\varepsilon_{4} e^{\varrho \tau} \leq 0$. By substituting (31) into (30), we obtain

$$
\begin{aligned}
D^{+} U^{*}\left(t^{*}\right) & <\left(\varrho-\bar{\varepsilon}_{3}+\varepsilon_{4} e^{\varrho \tau}\right) e^{\varrho t^{*}} V\left(t^{*}\right)+d e^{\varrho t^{*}}+\lambda_{\max }(Q) e^{\varrho t^{*}}\left|v\left(t^{*}\right)\right|^{2} \\
& \leq e^{\varrho t^{*}}\left(d+\lambda_{\max }(Q)\left|v\left(t^{*}\right)\right|^{2}\right)
\end{aligned}
$$

This conclusion contradicts (29), which indicates (25) is true.

For the purpose of RISpS property of system (7), we deduce from (25) that

$$
\begin{aligned}
V(t) & \leq \lambda_{\max }(P)|\varphi|_{\tau}^{2} e^{-\varrho t}+\int_{0}^{t} e^{-\varrho(t-s)}\left(d+\lambda_{\max }(Q)|v(s)|^{2}\right) d s \\
& \leq \lambda_{\max }(P)|\varphi|_{\tau}^{2} e^{-\varrho t}+\int_{0}^{t} e^{-\varrho(t-s)}\left(d+\lambda_{\max }(Q)|v|_{\infty}^{2}\right) d s \\
& \leq \lambda_{\max }(P)|\varphi|_{\tau}^{2} e^{-\varrho t}+\frac{d+\lambda_{\max }(Q)|v|_{\infty}^{2}}{\varrho} .
\end{aligned}
$$

According to Lemma 2, one derives

$$
|x(t)|^{2} \leq \frac{\lambda_{\max }(P)}{\lambda_{\min }(P)}|\varphi|_{\tau}^{2} e^{-\varrho t}+\frac{d+\lambda_{\max }(Q)|\nu|_{\infty}^{2}}{\lambda_{\min }(P) \varrho}
$$

which gives

$$
|x(t)| \leq \sqrt{\frac{\lambda_{\max }(P)}{\lambda_{\min }(P)}}|\varphi|_{\tau} e^{-\frac{\varrho}{2} t}+\sqrt{\frac{\lambda_{\max }(Q)}{\lambda_{\min }(P) \varrho}}|\nu|_{\infty}+\sqrt{\frac{d}{\lambda_{\min }(P) \varrho}} .
$$


By denoting $\beta_{c}(s, t)=\sqrt{\frac{\lambda_{\max }(P)}{\lambda_{\min }(P)}} s e^{-\frac{\varrho}{2} t}, \gamma_{c}(s)=\sqrt{\frac{\lambda_{\max }(Q)}{\lambda_{\min }(P) \varrho}} S$, and $d_{c}=\sqrt{\frac{\lambda_{\max }\left(P^{T} P\right) \xi_{2}}{\varrho\left(1-2 \xi_{1}\right) \lambda_{\min }(P)}}$, it is easily concluded that

$$
|x(t)| \leq \beta_{c}\left(|\varphi|_{\tau}, t\right)+\gamma_{c}\left(|v|_{\infty}\right)+d_{c}
$$

which means the system (7) is RISpS. The proof is complete.

Remark 4 It follows from (34) that the states of closed-loop system will eventually enter the set $\mathcal{B}\left(0, \gamma_{c}\left(|v|_{\infty}\right)+d_{c}\right)$ bounded by the threshold parameter $\xi_{2}$ and the exogenous disturbance $|v|_{\infty}$. When $\xi_{2}=0$, the states $x(t)$ converge to the zero with the exponential convergence rate $\varrho$ determined by $\varrho-\bar{\varepsilon}_{3}+\varepsilon_{4} e^{\varrho \tau} \leq 0$ if the exogenous disturbance decays to zero. If the exogenous disturbance $v(t)=0$, then the boundary of $\mathcal{B}\left(0, \gamma_{c}\left(|v|_{\infty}\right)+d_{c}\right)$ can be arbitrarily small if the threshold parameter $\xi_{2}$ is designed appropriately.

Remark 5 Theorem 1 provides an effective method to investigate the RISpS property of the closed-loop system (7) accounting for all admissible parameter uncertainties, which can be considered as an extension of Theorem 1 in [34]. The RISpS property means that state of the closed-loop system (7) will eventually enter the set $\mathcal{B}\left(0, \gamma_{c}\left(|v|_{\infty}\right)+d_{c}\right)$ bounded by the threshold parameter $\xi_{2}$ and the exogenous disturbance $|v|_{\infty}$. This dynamical performance is quite different from and more general than the asymptotical stability $[6,14]$, robust stability [16-27], and the conventional ISS [10, 12, 13]. Furthermore, from the point of view of technique analysis, we adopt the Lyapunov function method for the dynamics of state $x(t)$ while the impulsive jumping estimation method for the control input $u\left(t_{k}\right)$ at event-triggering instants, which shows some hybrid characteristics and is quite different from the common L-K functional approach used in [16-18, 21-27, 48].

It should be pointed out that the Zeno behavior, which means the controller is triggered infinitely in a limited time interval, will seriously impact the operation of sampling devices and must therefore be excluded.

Theorem 2 If all conditions of Theorem 1 hold, then the Zeno behavior does not exist for the closed-loop system (7).

Proof Recalling $e(t)=x\left(t_{i}\right)-x(t)$, we calculate the upper right-hand Dini derivative of $|e(t)|^{2}$ for $t \in\left[t_{i}, t_{i+1}\right)$ as follows:

$$
\begin{aligned}
D^{+}|e(t)|^{2}= & -2 e^{T}(t)\left[(A+\Delta A+B K) x(t)+\left(A_{d}+\Delta A_{d}\right) x_{\tau}(t)+B K e(t)+C v(t)\right] \\
= & -2 e^{T}(t)\left[(A+\Delta A+B K)\left(x\left(t_{i}\right)-e(t)\right)+\left(A_{d}+\Delta A_{d}\right) x_{\tau}(t)+B K e(t)+C v(t)\right] \\
= & 2 e^{T}(t)(A+\Delta A) e(t)-2 e^{T}(t) C v(t)-2 e^{T}(t)(A+\Delta A+B K) x\left(t_{i}\right) \\
& -2 e^{T}(t)\left(A_{d}+\Delta A_{d}\right) x_{\tau}(t) .
\end{aligned}
$$

It follows from the inequality $2 a^{T} J b \leq\|J\|\left(|a|^{2}+|b|^{2}\right)$ that

$$
\begin{aligned}
D^{+}|e(t)|^{2} \leq & \left(2\|A+\Delta A\|+\left\|A_{d}+\Delta A_{d}\right\|\right. \\
& +\|A+\Delta A+B K\|+\|C\|)|e(t)|^{2}
\end{aligned}
$$




$$
\begin{aligned}
& +\|A+\Delta A+B K\|\left|x\left(t_{i}\right)\right|^{2} \\
& +\left\|A_{d}+\Delta A_{d}\right\|\left|x_{\tau}(t)\right|^{2}+\|C\||v(t)|^{2} \\
\leq & a_{1}|e(t)|^{2}+a_{2}\left|x\left(t_{i}\right)\right|^{2}+a_{3}\left|x_{\tau}(t)\right|^{2}+|\|C\| v(t)|^{2}
\end{aligned}
$$

in which $a_{1}=3\|A\|+3\left\|E_{1}\right\|\left\|H_{1}\right\|+\left\|A_{d}\right\|+\left\|E_{2}\right\|\left\|H_{2}\right\|+\|B\|\|K\|+\|C\|, a_{2}=\|A\|+$ $\left\|E_{1}\right\|\left\|H_{1}\right\|+\|B\|\|K\|$, and $a_{3}=\left\|A_{d}\right\|+\left\|E_{2}\right\|\left\|H_{2}\right\|$.

According to (33), we derive that

$$
\begin{aligned}
\left|x\left(t_{i}\right)\right|^{2} & \leq \frac{\lambda_{\max }(P)}{\lambda_{\min }(P)}|\varphi|_{\tau}^{2} e^{-\varrho t_{i}}+\frac{d+\lambda_{\max }(Q)|v|_{\infty}^{2}}{\lambda_{\min }(P) \varrho} \\
& \leq \frac{\lambda_{\max }(P)}{\lambda_{\min }(P)}|\varphi|_{\tau}^{2}+\frac{d+\lambda_{\max }(Q)|v|_{\infty}^{2}}{\lambda_{\min }(P) \varrho}:=M_{1}
\end{aligned}
$$

and

$$
\begin{aligned}
\left|x_{\tau}(t)\right|^{2} & \leq \frac{\lambda_{\max }(P)}{\lambda_{\min }(P)}|\varphi|_{\tau}^{2} e^{-\varrho(t-\tau(t))}+\frac{d+\lambda_{\max }(Q)|v|_{\infty}^{2}}{\lambda_{\min }(P) \varrho} \\
& \leq \frac{\lambda_{\max }(P)}{\lambda_{\min }(P)}|\varphi|_{\tau}^{2} e^{\varrho \tau}+\frac{d+\lambda_{\max }(Q)|\nu|_{\infty}^{2}}{\lambda_{\min }(P) \varrho}:=M_{2} .
\end{aligned}
$$

Substituting (38) and (39) into (37), one has

$$
D^{+}|e(t)|^{2} \leq a_{1}|e(t)|^{2}+a_{2} M_{1}+a_{3} M_{2}+\|C\||v|^{2} .
$$

Letting $\bar{M}=a_{2} M_{1}+a_{3} M_{2}+\|C\||v|^{2}$ and multiplying both sides of (40) by $e^{-a_{1}\left(t-t_{i}\right)}$ leads to

$$
e^{-a_{1}\left(t-t_{i}\right)} D^{+}|e(t)|^{2} \leq a_{1} e^{-a_{1}\left(t-t_{i}\right)}|e(t)|^{2}+\bar{M} e^{-a_{1}\left(t-t_{i}\right)},
$$

which implies that

$$
D^{+}\left(e^{-a_{1}\left(t-t_{i}\right)}|e(t)|^{2}\right) \leq \bar{M} e^{-a_{1}\left(t-t_{i}\right)} .
$$

Noting that $e\left(t_{i}\right)=0$, it is readily deduced that

$$
|e(t)|^{2} \leq \int_{t_{i}}^{t} \bar{M} e^{a_{1}(t-s)} d s=\frac{\bar{M}}{a_{1}}\left(e^{a_{1}\left(t-t_{i}\right)}-1\right) .
$$

Bearing in mind that the event-triggered strategy (4) indicates the control updating will not occur until $|e(t)|^{2}=\xi_{1}\left|x\left(t_{i}\right)\right|^{2}+\xi_{2}$, we deduce that the next triggering instant $t_{i+1}$ satisfies

$$
\xi_{1}\left|x\left(t_{i}\right)\right|^{2}+\xi_{2} \leq \frac{\bar{M}}{a_{1}}\left(e^{a_{1}\left(t_{i+1}-t_{i}\right)}-1\right) .
$$

That is to say

$$
t_{i+1}-t_{i} \geq \frac{1}{a_{1}} \ln \left(1+\frac{a_{1}\left(\xi_{1}\left|x\left(t_{i}\right)\right|^{2}+\xi_{2}\right)}{\bar{M}}\right) .
$$

Therefore, we exclude the Zeno behavior. 
Remark 6 In [32, 33, 40, 41], the ratio $\frac{|e(t)|}{|x(t)|}$ is estimated to exclude the Zeno behavior caused by the event-triggered control for continuous-time systems. It should be noted that this technique will be invalid for event-triggered scheme (4) because of the presence of time delays, parameter uncertainties as well as exogenous disturbances. To this end, a hybrid analysis method is employed to overcome this difficulty. To be specific, we first obtain the upper bounds for the delayed state and the jump state at event-triggering time. Then the evolution of the measurement error $e(t)$ is derived with the help of a differential comparison system with a nonnegative bound. Finally, the Zeno behavior is excluded for the proposed event-triggered scheme while the RISpS property is maintained for the closed-loop system (7).

Now, we are ready to design the feedback gain matrix $K$ and the event-triggered parameters $\xi_{1}, \xi_{2}$ for the controller.

Theorem 3 If there exist two positive definite matrices $\tilde{P} \in \mathbb{R}^{n \times n}, \tilde{Q} \in \mathbb{R}^{n \times n}$, a constant matrix $Y \in \mathbb{R}^{q \times n}$, and four positive scalars $\varepsilon_{1}, \varepsilon_{2}$ and $\tilde{\varepsilon_{3}}>\tilde{\varepsilon_{4}}$ such that

$$
\begin{aligned}
& I-\varepsilon_{2} E_{2} E_{2}^{T}>0, \\
& \left(\begin{array}{ccc}
\tilde{\Pi}_{11}^{*} & \tilde{\Pi}_{12}^{*} & \tilde{\Pi}_{13}^{*} \\
* & -W & 0 \\
* & * & -\varepsilon_{2} I
\end{array}\right)<0,
\end{aligned}
$$

and

$$
\left(\begin{array}{cccc}
\tilde{\Pi}_{11} & \tilde{\Pi}_{12} & \tilde{\Pi}_{13} & \tilde{\Pi}_{14} \\
* & -I & 0 & 0 \\
* & * & -\tilde{Q} & 0 \\
* & * & * & -\varepsilon_{1} I
\end{array}\right)<0
$$

where $\tilde{\Pi}_{11}^{*}=-\tilde{\varepsilon}_{4} \tilde{P}, \tilde{\Pi}_{12}^{*}=\tilde{P} A_{d}^{T}, \tilde{\Pi}_{13}^{*}=\tilde{P} H_{2}^{T}, W=I-\varepsilon_{2} E_{2} E_{2}^{T}, \tilde{\Pi}_{11}=A \tilde{P}+\tilde{P} A^{T}+\tilde{\varepsilon_{3}} \tilde{P}+B Y+$ $Y^{T} B^{T}+I+\varepsilon_{1} E_{1} E_{1}^{T}, \tilde{\Pi}_{12}=B Y, \tilde{\Pi}_{13}=C, \tilde{\Pi}_{14}=\tilde{P} H_{1}^{T}$, then the closed-loop system (7) is robustly input-to-state practically stable and the event-triggered controller is designed with the gain matrix satisfying

$$
K=Y \tilde{P}^{-1}
$$

and the event-triggered parameters $\xi_{1}$ and $\xi_{2}$ satisfying

$$
0<\xi_{1}<\frac{\left(\tilde{\varepsilon_{3}}-\tilde{\varepsilon_{4}}\right) \lambda_{\min }\left(\tilde{P}^{-1}\right)}{2\left(\lambda_{\max }\left(\tilde{P}^{-2}\right)+\left(\tilde{\varepsilon_{3}}-\tilde{\varepsilon}_{4}\right) \lambda_{\min }\left(\tilde{P}^{-1}\right)\right)}, \quad \xi_{2}>0
$$

Proof Pre- and post-multiplying (46) and (47) by $\operatorname{diag}\left\{\tilde{P}^{-1}, I, I\right\}$ and $\operatorname{diag}\left\{\tilde{P}^{-1}, I, I, I\right\}$, respectively, we obtain

$$
\left(\begin{array}{ccc}
-\tilde{\varepsilon}_{4} \tilde{P}^{-1} & A_{d}^{T} & H_{2}^{T} \\
* & -W & 0 \\
* & * & -\varepsilon_{2} I
\end{array}\right)<0
$$


and

$$
\left(\begin{array}{cccc}
\tilde{P}^{-1} \tilde{\Pi}_{11} \tilde{P}^{-1} & \tilde{P}^{-1} B Y & \tilde{P}^{-1} C & H_{1}^{T} \\
* & -I & 0 & 0 \\
* & * & -\tilde{Q} & 0 \\
* & * & * & -\varepsilon_{1}^{-1} I
\end{array}\right)<0 .
$$

By using the Schur complement formula, we conclude that

$$
A_{d}^{T}\left(I-\varepsilon_{2} E_{2} E_{2}^{T}\right)^{-1} A_{d}+\varepsilon_{2}^{-1} H_{2}^{T} H_{2}-\tilde{\varepsilon_{4}} \tilde{P}^{-1}<0
$$

and

$$
\begin{aligned}
& \tilde{P}^{-1} A+A^{T} \tilde{P}^{-1}+\tilde{P}^{-1} B K+K^{T} B^{T} \tilde{P}^{-1}+\tilde{P}^{-1} C \tilde{Q}^{-1} C^{T} \tilde{P}^{-1}+\tilde{P}^{-1} \tilde{P}^{-1} \\
& +\tilde{P}^{-1} B K \tilde{P}^{2} K^{T} B^{T} \tilde{P}^{-1}+\varepsilon_{1} H_{1}^{T} H_{1}+\varepsilon_{1}^{-1} \tilde{P}^{-1} E_{1} E_{1}^{T} \tilde{P}^{-1}+\tilde{\varepsilon}_{3} \tilde{P}^{-1}<0 .
\end{aligned}
$$

Letting $\tilde{\varepsilon_{3}}=\varepsilon_{3}, \tilde{\varepsilon_{4}}=\varepsilon_{4}, \tilde{P}^{-1}=P$ and $\tilde{Q}=Q$, one derives (9) and (10). In addition, it is readily deduced from (45) and (49) that (8) and (11) hold, respectively. The proof is complete.

Remark 7 Theorem 3 provides a design method for the event-triggered feedback controller which is employed to ensure the RISpS for the closed-loop system (7). According to the feasibility of (45)-(49), the gain matrix $K$, the weight parameter $\xi_{1}$, and the threshold parameter $\xi_{2}$ are co-designed. The proposed controller is more practical than those with pre-fixed triggered parameters. Furthermore, the event-triggered feedback controller in Theorem 3 is delicately designed to explain some robustness with regard to the time delays and parameter uncertainties, whose effects have already been included in the inequalities (45)-(47).

Remark 8 The parameters of controller are directly calculated according to the feasible solutions of (45)-(49) which can be easily solved with the help of Matlab LMI toolbox. That means our result is feasible and computable. In addition, it is readily observed that the linear matrix inequalities (LMIs) (45)-(49) have smaller dimensions and fewer variables than those given in [16-27], which leads to a lower computation complexity.

\section{A numerical example}

In this section, a numerical example is provided to show the effectiveness of proposed results. Consider the following system parameters:

$$
\begin{aligned}
& A=\left(\begin{array}{cc}
-1.7 & -0.7 \\
-0.4 & -1.95
\end{array}\right), \quad A_{d}=\left(\begin{array}{cc}
0.3 & 0.5 \\
-0.2 & 0.3
\end{array}\right), \quad B=\left(\begin{array}{c}
-1.2 \\
1.3
\end{array}\right), \\
& C=\left(\begin{array}{cc}
1.6 & 2.1 \\
-2.2 & 1.3
\end{array}\right), \quad E_{1}=\left(\begin{array}{l}
1.8 \\
1.6
\end{array}\right), \quad H_{1}=\left(\begin{array}{ll}
0.1 & 0.3
\end{array}\right), \\
& E_{2}=\left(\begin{array}{l}
1.0 \\
0.9
\end{array}\right), \quad H_{2}=\left(\begin{array}{ll}
0.8 & 1.4
\end{array}\right) .
\end{aligned}
$$


Figure 1 The state of uncertain delayed system without exogenous disturbances

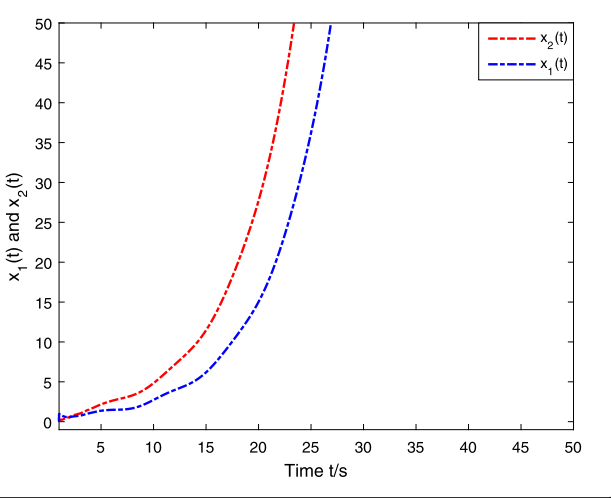

It is worth noting that the open-loop system with above parameters is unstable even though the exogenous disturbance is absent. The simulation result is illustrated in Fig. 1.

Let $\tilde{\varepsilon}_{3}=1.98$ and $\tilde{\varepsilon}_{4}=1.21$. By employing the Matlab toolbox to solve inequalities (45)(47), we obtain a set of feasible solutions as follows:

$$
\begin{aligned}
& \tilde{P}=\left(\begin{array}{cc}
2.5030 & -0.8972 \\
-0.8972 & 0.4759
\end{array}\right), \quad \tilde{Q}=10^{6} \times\left(\begin{array}{cc}
9.7138 & 0.0338 \\
0.0338 & 9.6605
\end{array}\right), \\
& Y=\left(\begin{array}{ll}
-0.0748 & -0.8384
\end{array}\right) .
\end{aligned}
$$

The upper bound of event-triggered weight parameter is calculated to be

$$
\frac{\left(\tilde{\varepsilon}_{3}-\tilde{\varepsilon}_{4}\right) \lambda_{\min }\left(\tilde{P}^{-1}\right)}{2\left(\lambda_{\max }\left(\tilde{P}^{-2}\right)+\left(\tilde{\varepsilon}_{3}-\tilde{\varepsilon}_{4}\right) \lambda_{\min }\left(\tilde{P}^{-1}\right)\right)}=0.0025
$$

which means $0<\xi_{1}<0.0025$ and $\xi_{2}>0$. According to Theorem 3, we select the eventtriggered parameters $\xi_{1}=0.002, \xi_{2}=0.1$, and the feedback gain matrix

$$
K=Y \tilde{P}^{-1}=\left(\begin{array}{ll}
-2.0398 & -5.6070
\end{array}\right)
$$

to design the controller for ensuring the desired dynamical performance.

For the aim of simulation, we choose the time interval $[0,50 s]$ and the step $0.001 s$. Moreover, the unknown-but-bounded exogenous disturbance $v(t)$ is chosen to be $v_{1}(t)=$ $v_{0} \sin (t)$ and $v_{2}(t)=v_{0} \cos (t)$ where $v_{0}$ is a set of randomly generated numbers in the interval $(-0.1,0.1)$. The initial value is selected to be 1 . The simulation results for control performance are shown in Fig. 2 and Fig. 3. To be specific, the state evolution of closedloop system is shown in Fig. 2 from which we see that the state $x(t)$ enters a bounded set under the event-triggered control input. Figure 3(a) presents the response of control signals and the curve shows that $u(t)$ keeps as a constant between two consecutively triggered instants. Figure 3(b) depicts the event-triggered instants and the control signal releasing intervals, which implies the frequency of control updating is greatly reduced.

Therefore, it is confirmed from the simulation results that the closed-loop system with the proposed event-triggered feedback controller is robustly input-to-state practically stable. 
Figure 2 The state evolution of the closed-loop system

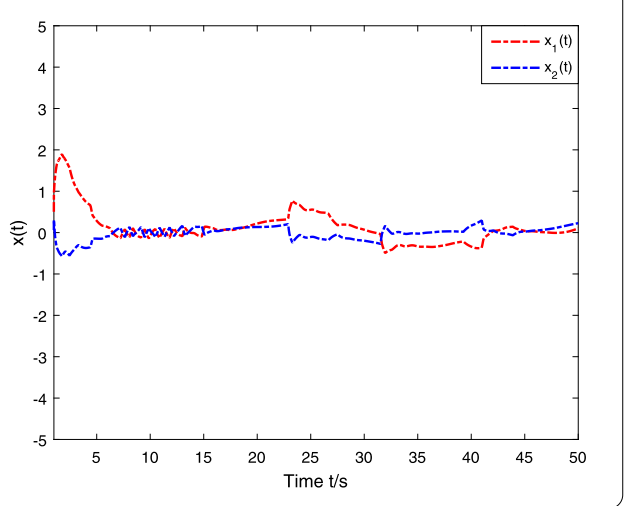

Figure 3 (a) The response of control input $u(t)$. (b) The event-triggered instants
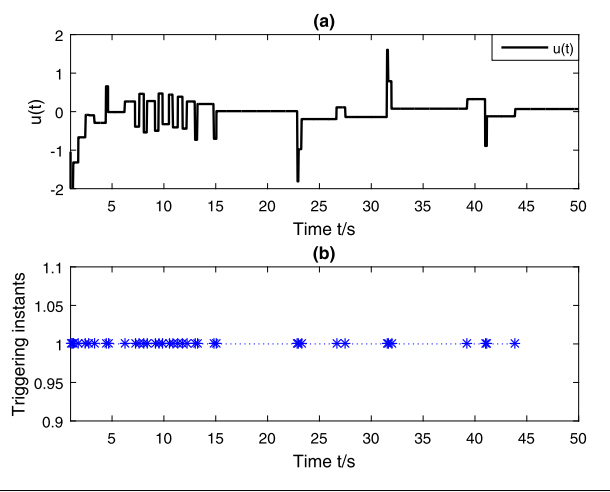

Remark 9 In [16-27], some interesting results have been derived (in the form of LMIs) to address the robust stability for neural networks with both delays and parameter uncertainties. However, these results are invalid to our Example due mainly to the present of the bounded exogenous disturbances and the sporadic event-based control input. Moreover, it is obviously seen that the approaches given in [29-33, 36] cannot be used to investigate the dynamical behavior and analyze the Zeno phenomenon for Example 1 because of the coupled effects from time delays, parameter uncertainties as well as hybrid event-triggered scheme.

\section{Conclusions}

In this paper, the RISpS problem for a class of uncertain delayed systems with exogenous disturbances has been investigated. An event-triggered strategy has been introduced to effectively reduce the updating frequency for the robust controller. Several criteria have been established to address the RISpS property for the closed-loop system and the controller design. In particular, the Zeno behavior has been analyzed and excluded by utilizing the information of current and delayed states, parameter uncertainties, and exogenous disturbances. Finally, a numerical example has been given to illustrate the effectiveness of our results. 


\section{Funding}

This work was supported in part by the National Natural Science Foundation of China under Grant 61773004, the Natural Science Foundation of Chongqing under Grant cstc2019jcyj-msxmX0722, the Program of Chongqing Innovation Team Project in University under Grant CXTDX201601022, the Science and Technology Research Program of Chongqing Municipal Education Commission under Grant KJQN201800733, the Innovation Project for Returned Overseas Scholars of Chongqing under Grant CX2018115, and the Research Innovation Project for Postgraduate of Chongqing Jiaotong University under Grant 2019501204

\section{Competing interests}

The authors declare that they have no competing interests.

Authors' contributions

All authors conceived of the study, participated in its design and coordination, read and approved the final manuscript.

\section{Authors' information}

Y. Du and B. Li are with the Department of Mathematics, Chongqing Jiaotong University, Chongqing 400074, China.

\section{Publisher's Note}

Springer Nature remains neutral with regard to jurisdictional claims in published maps and institutional affiliations.

Received: 7 April 2019 Accepted: 12 December 2019 Published online: 21 February 2020

\section{References}

1. Stamova, I., Stamov, G.: Mittag-Leffler synchronization of fractional neural networks with time-varying delays and reaction-diffusion terms using impulsive and linear controllers. Neural Netw. 96, 22-32 (2017)

2. Stamova, I., Stamov, G., Simeonov, S., Ivanov, A.: Mittag-Leffler stability of impulsive fractional-order bi-directional associative memory neural networks with time-varying delays. Trans. Inst. Meas. Control 40, 3068-3077 (2018)

3. Yang, D., Li, X., Qiu, J.: Output tracking control of delayed switched systems via state-dependent switching and dynamic output feedback. Nonlinear Anal. Hybrid Syst. 32, 294-305 (2019)

4. Li, X., Yang, X., Huang, T.: Persistence of delayed cooperative models: impulsive control method. Appl. Math. Comput. 342, 130-146 (2019)

5. Yang, X., Li, X., Xi, Q., Duan, P.: Review of stability and stabilization for impulsive delayed systems. Math. Biosci. Eng. $15(6), 1495-1515(2018)$

6. Ma, L., Wang, Z., Liu, Y., Alsaadi, F.E.: Exponential stabilization of nonlinear switched systems with distributed time-delay: an average dwell time approach. Eur. J. Control 37, 34-42 (2017)

7. Sontag, E.: Smooth stabilization implies coprime factorization. IEEE Trans. Autom. Control 34(4), 435-443 (1989)

8. Gao, L., Wang, D.: Input-to-state stability and integral input-to-state stability for impulsive switched systems with time-delay under asynchronous switching. Nonlinear Anal. Hybrid Syst. 20, 55-71 (2016)

9. Liu, B., Hill, D.J., Sun, Z.: Input-to-state-KL-stability and criteria for a class of hybrid dynamical systems. Appl. Math. Comput. 326, 124-140 (2018)

10. Liu, K., Fridman, E., Johansson, K.H.: Exponential input-to-state stability under events for hybrid dynamical networks with coupling time-delays. Automatica 59, 248-255 (2015)

11. Mironchenko, A., Wirth, F.: Lyapunov characterization of input-to-state stability for semilinear control systems over Banach spaces. Syst. Control Lett. 119,64-70 (2018)

12. Ning, C., He, Y., Wu, M., Zhou, S.: Indefinite Lyapunov functions for input-to-state stability of impulsive systems. Inf. Sci. 436, 343-351 (2018)

13. Sun, F., Gao, L., Zhu, W., Liu, F.: Generalized exponential input-to-state stability of nonlinear systems with time delay. Commun. Nonlinear Sci. Numer. Simul. 44, 352-359 (2017)

14. Huang, L., Mao, X.: On input-to-state stability of stochastic retarded systems with Markovian switching. IEEE Trans. Autom. Control 54(8), 1898-1902 (2009)

15. Zhao, Y., Kurths, J., Duan, L.: Input-to-state stability analysis for memristive BAM neural networks with variable time delays. Phys. Lett. A 383(11), 1143-1150 (2019)

16. Wang, Z., Liu, Y., Liu, X.: $H_{\infty}$ filtering for uncertain stochastic time-delay systems with sector-bounded nonlinearities Automatica 44, 1268-1277 (2008)

17. Liu, K., Friman, E., Johansson, K.H.: Dynamic quantization of uncertain linear networked control systems. Automatica 59, 248-255 (2015)

18. Sowmiya, C., Raja, R., Cao, J., Rajchakit, G., Alsaedi, A.: Enhanced robust finite-time passivity for Markovian jumping discrete-time BAM neural networks with leakage delay. Adv. Differ. Equ. 2017, 318 (2017)

19. Shen, B., Wang, Z., Tan, H.: Guaranteed cost control for uncertain nonlinear systems with mixed time-delays: the discrete-time case. Eur. J. Control 40, 62-67 (2018)

20. Liu, Y., Wang, Z., Ma, L., Alsaadi, F.E.: Robust $H_{\infty}$ control for a class of uncertain nonlinear systems with mixed time-delays. J. Franklin Inst. 355(14), 6339-6352 (2018)

21. Maharajan, C., Raja, R., Cao, J., Rajchakit, G.: Novel global robust exponential stability criterion for uncertain inertial-type BAM neural networks with discrete and distributed time-varying delay via Lagrange sense. J. Franklin Inst. 355(11), 4727-4754 (2018)

22. Pandiselvi, S., Raja, R., Cao, J., Rajchakit, G., Ahmad, B.: Approximation of state variables for discrete-time stochastic genetic regulatory networks with leakage, distributed, and probabilistic measurement delays: a robust stability problem. Adv. Differ. Equ. 2018, 123 (2018)

23. Saravanakumar, R., Rajchakit, G., Ali, M., Xiang, Z., Joo, Y.: Robust extended dissipativity criteria for discrete-time uncertain neural networks with time-varying delays. Neural Comput. 30(12), 3893-3904 (2018) 
24. Maharajan, C., Raja, R., Cao, J., Ravi, G., Rajchakit, G.: Global exponential stability of Markovian jumping stochastic impulsive uncertain BAM neural networks with leakage, mixed time delays, and $\alpha$-inverse Hölder activation functions. Adv. Differ. Equ. 2018, 113 (2018)

25. Sowmiya, C., Raja, R., Cao, J., Rajchakit, G.: Enhanced result on stability analysis of randomly occurring uncertain parameters, leakage, and impulsive BAM neural networks with time-varying delays: discrete-time case. Int. J. Adapt. Control Signal Process. 32(7), 1010-1039 (2018)

26. Sowmiya, C., Raja, R., Cao, J., Rajchakit, G., Alsaedi, A.: Exponential stability of discrete-time cellular uncertain BAM neural networks with variable delays using Halanay-type inequality. Appl. Math. Inf. Sci. 12(3), 545-558 (2018)

27. Chandran, S., Ramachandran, R., Cao, J., Agarwal, R., Rajchakit, G.: Passivity analysis for uncertain BAM neural networks with leakage, discrete and distributed delays using novel summation inequality. Int. J. Control. Autom. Syst. 17(8), 2114-2124 (2019)

28. Liu, Q., Wang, Z., He, X., Zhou, D.H.: A survey of event-based strategies on control and estimation. Syst. Sci. Control Eng. 2(1), 90-97 (2014)

29. Persis, C., Sailer, R., Wirth, F.: Parsimonious event-triggered distributed control: a zeno free approach. Automatica 49(7), 2116-2124 (2013)

30. Tabuada, P.: Event-triggered real-time scheduling of stabilizing control tasks. IEEE Trans. Autom. Control 52(9), 1680-1685 (2007)

31. Wang, X., Lemmon, M.: Event-triggered broadcasting across distributed networked control systems. In: Proceedings of the 2008 American Control Conference, Washington, USA, pp. 3139-3144 (2008)

32. Lunze, J., Lehmann, D.: A state-feedback approach to event-based control. Automatica 46(1), $211-215$ (2010)

33. Garcia, E., Antsaklis, P.: Model-based event-triggered control for systems with quantization and time-varying network delays. IEEE Trans. Autom. Control 58(2), 422-434 (2013)

34. Li, B., Wang, Z., Han, Q.-L.: Input-to-state stabilization of delayed differential systems with exogenous disturbances: the event-triggered case. IEEE Trans. Syst. Man Cybern. Syst. 49(6), 1099-1109 (2019)

35. Zhang, P., Liu, T., Jiang, Z.-P.: Input-to-state stabilization of nonlinear discrete-time systems with event-triggered controllers. Syst. Control Lett. 103, 16-22 (2017)

36. $\mathrm{Yu}, \mathrm{H} ., \mathrm{Hao}$, F.: Input-to-state stability of integral-based event-triggered control for linear plants. Automatica 85 , 248-255 (2017)

37. Shen, B., Wang, Z., Qiao, H.: Event-triggered state estimation for discrete-time multidelayed neural networks with stochastic parameters and incomplete measurements. IEEE Trans. Neural Netw. Learn. Syst. 28(5), 1152-1163 (2017)

38. Sheng, L., Wang, Z., Zou, L., Alsaadi, F.: Event-based $H_{\infty}$ state estimation for time-varying stochastic dynamical networks with state-and disturbance-dependent noises. IEEE Trans. Neural Netw. Learn. Syst. 28(10), 2382-2394 (2017)

39. Wang, Z., Hu, J., Ma, L.: Event-based distributed information fusion over sensor networks. Inf. Fusion 39, 53-55 (2018)

40. Dimarogonas, D., Frazzoli, E., Johansson, K.: Distributed event-triggered control for multi-agent systems. IEEE Trans. Autom. Control 57(5), 1291-1297 (2012)

41. Zhu, W., Jiang, Z.-P.: Event-based leader-following consensus of multi-agent systems with input time delay. IEEE Trans Autom. Control 60(5), 1362-1367 (2015)

42. Abdelrahim, M., Postoyan, R., Daafouz, J., Nešić, D.: Robust event-triggered output feedback controllers for nonlinear systems. Automatica 75, 96-108 (2017)

43. Yang, J., Sun, J., Zheng, W., Li, S.: Periodic event-triggered robust output feedback control for nonlinear uncertain systems with time-varying disturbance. Automatica 94, 324-333 (2018)

44. Liu, D., Yang, G.-H.: Robust event-triggered control for networked control systems. Inf. Sci. 459, 186-197 (2018)

45. Zhang, P., Liu, T., Jiang, Z.-P.: Robust event-triggered control subject to external disturbance. IFAC-PapersOnLine 50(1), 7899-7904 (2017)

46. Ma, R., Shao, X., Liu, J., Wu, L.: Event-triggered sliding mode control of Markovian jump systems against input saturation. Syst. Control Lett. (2019). https://doi.org/10.1016/j.sysconle.2019.104525

47. Guan, Z.-H., Hill, D.J., Shen, X.: Input-to-state stabilization of nonlinear discrete-time systems with event-triggered controllers. IEEE Trans. Autom. Control 50(7), 1058-1062 (2005)

48. Wang, Y., Xie, L., Souza, C.E.: Robust control of a class of uncertain nonlinear system. Syst. Control Lett. 19, 139-149 (1992)

\section{Submit your manuscript to a SpringerOpen ${ }^{\circ}$ journal and benefit from:}

- Convenient online submission

- Rigorous peer review

- Open access: articles freely available online

- High visibility within the field

- Retaining the copyright to your article

Submit your next manuscript at $>$ springeropen.com 